\title{
Design and Analysis of An Active Comb Filter based on Low Transconductance OTA-C for Biomedical Applications
}

\author{
Arvind Kumar Sahu' ${ }^{1}$ Anil Kumar Sahu² \\ ${ }^{1}$ ME IV Semester, Department of ET\&T, FET, SSTC, Bhilai \\ ${ }^{2}$ Assistant Professor, Department of ET\&T, FET, SSTC, Bhilai
}

\begin{abstract}
The design of a OTA-C based comb filter is presented in this paper. The comb filter is designed by using only OTA and capacitors, which can be easily fabricated into an IC. The filter was implemented in Tanner EDA tool using 250nm technology. The simulation result shows that this filter was able to provide stop band attenuation of $20 \mathrm{db}$ for $50 \mathrm{~Hz}, 150 \mathrm{~Hz}$ and $250 \mathrm{~Hz}$, powerline harmonics.
\end{abstract}

Keywords: Biomedical signal, Comb filter, OTA-C, powerline interference

\section{Introduction}

Measuring bio-potential signals, such as Electroencephalogram (EEG), Electrocardiogram (ECG), and Electromyogram (EMG) against various contaminating signals in portable biomedical monitoring systems is of great challenge in minimizing the power consumption while maintaining a high performance. The voltage levels of various biomedical signals are given in Table 1[3].

Table 1: Properties of Physiological Signals

\begin{tabular}{|c|c|}
\hline Physiological Signal & Measurement Range \\
\hline Electroencephalography (EEG) & $25-300 \mu \mathrm{V}$ \\
\hline Electroretinogram (ERG) & $5-900 \mu \mathrm{V}$ \\
\hline Electrogastrogram (EGG) & $10-1000 \mu \mathrm{V}$ \\
\hline Electrocardiography (ECG) & $0.5-4 \mathrm{mV}$ \\
\hline Electromyography (EMG) & $0.1-1.5 \mathrm{mV}$ \\
\hline
\end{tabular}

The powerline interference $(50 \mathrm{~Hz} / 60 \mathrm{~Hz}$ and its harmonics) is greater than these biomedical signals [1]. In order to yield accurate readings from ECG/EEG there is a need to eliminate this interfering signal. These signals are also low frequency signals (well below the $1 \mathrm{KHz}$ mark for ECG, EEG, and ERG signals, and extending only up to 3 $\mathrm{KHz}$ for EMG signals). Therefore, it is observed from the literature that it has become cumbersome to record the electrical activity at such low frequencies. The on-chip implementation of filter circuits of this frequency range requires large time constants which implies use of large passive components (resistances and capacitances), which increases the size of wearable or implantable device, making it obtrusive.

These powerline interference may be removed by both analog and digital filters. Various methods of noise suppression using analog notch filter at frequency $50 \mathrm{~Hz} / 60$ $\mathrm{Hz}$ have been described [2]-[7].

In this paper an active comb filter using low transconductance OTA is proposed. This filter attenuates signal at $50 \mathrm{~Hz}$ and its odd harmonics at $150 \mathrm{~Hz}$ and $250 \mathrm{~Hz}$.
The stopband frequency and quality factor of filter can be easily tuned electronically by adjusting the bias voltage of OTAs.

\section{Methodology}

\subsection{RLC notch filter}

A simple second order passive notch filter consists of three elements, a resistor, a capacitor and an inductor as shown in Figure 1.

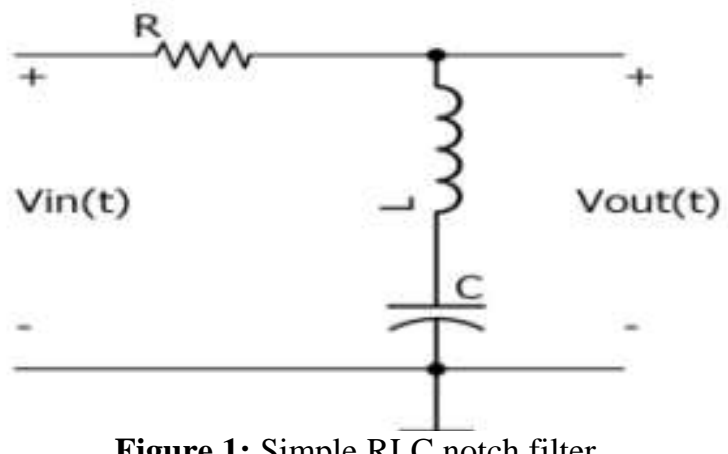

Figure 1: Simple RLC notch filter

The transfer function of the filter $\mathrm{H}(\mathrm{s})$ is given as

$$
H(s)=\frac{s^{2} L C+1}{s^{2} L C+s C R+1}
$$

The parameters of the filter are obtained as

$$
\begin{gathered}
\omega_{0}=\frac{1}{\sqrt{L C}} \\
Q_{0}=\frac{1}{R} \sqrt{\frac{L}{C}} \\
\Delta f=\frac{R}{L}
\end{gathered}
$$

A comb filter can be designed by using n-number of such notch filter with different center frequency to remove nnumber of harmonics.Comb filter using notch filter is shown in figure 2 . 


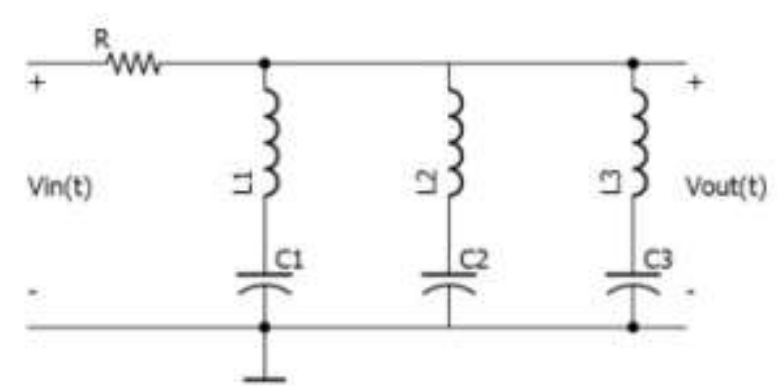

Figure 2: $\widehat{C o m b}$ filter using RLC notch filter

This comb filter using resistors, inductors and capacitors are too bulky to be fabricated in an IC. One way to eliminate inductors and large valued capacitors and resistors is to model inductors and resistors using active element such as operational transconductance amplifier (OTA).

\subsection{OTA}

OTA is similar to operational amplifiers (OPAMPS), both have differential input with infinite input impedances. The difference is in output, OPAMPS have voltage output with low output impedance and OTAs have current output with infinite output impedance. One advantage of OTA over OPAMP is the transconductance gain $\left(\mathrm{G}_{\mathrm{m}}\right)$ can be controlled electronically by adjusting bias current. The structure of OTA [8] used in this paper is a symmetrical cascade OTA as shown in figure 3. Symbolic representation of OTA is show in figure 4 .

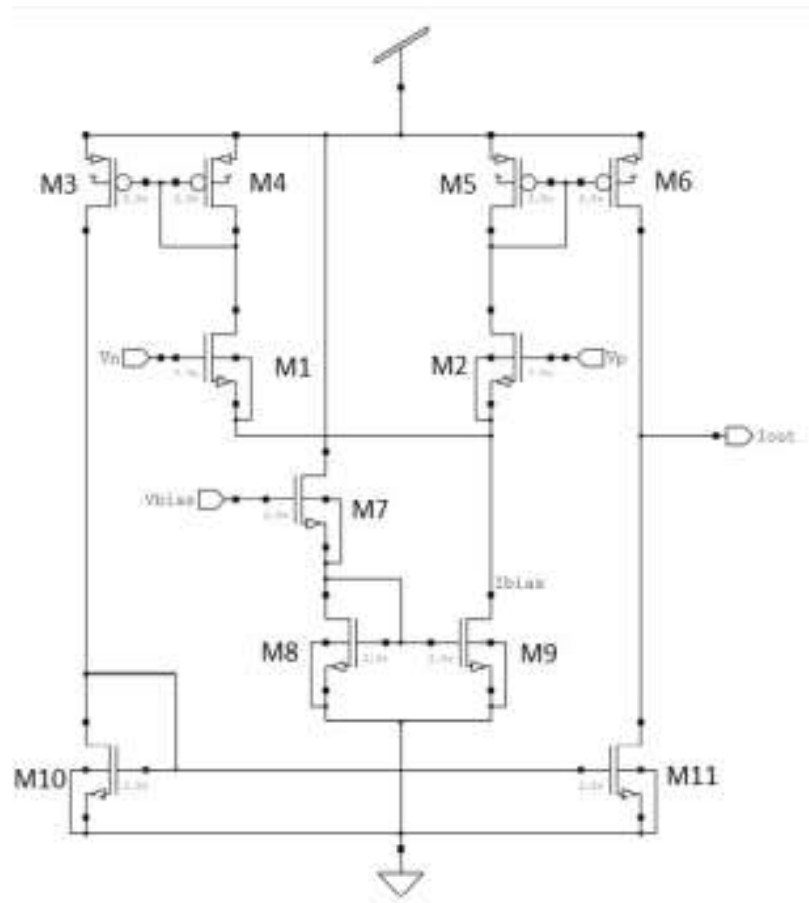

Figure 3: CMOS symmetricalcascade OTA

In the schematic of OTA, the differential input is take at gate of transistor M1 and M2. The bias current is controlled by applying bias voltage at the gate of transistor M7. And the $\mathrm{p}$ channel MOS transistor M3, M4, M5, M6 acts as active loads.

The relation between transconductance $\left(G_{m}\right)$ and bias current [8] is given as

$$
G_{m}=B \sqrt{2 \mu C_{O X}\left(\frac{W}{L}\right) I_{B}}
$$

where $\mathrm{B}$ is constant, $\mu$ is mobility of electron, $\mathrm{C}_{\mathrm{OX}}$ is oxide layer capacitance, $\mathrm{I}_{B}$ is bias current which can be controlled by controlling Vbias at the gate of transistor M7 in figure 3. Various characteristics of OTA such as variation of transconductance $\left(\mathrm{G}_{\mathrm{m}}\right)$ with frequency and variation of $\mathrm{G}_{\mathrm{m}}$ with Vbias is given in figure 5 and figure 6 respectively.

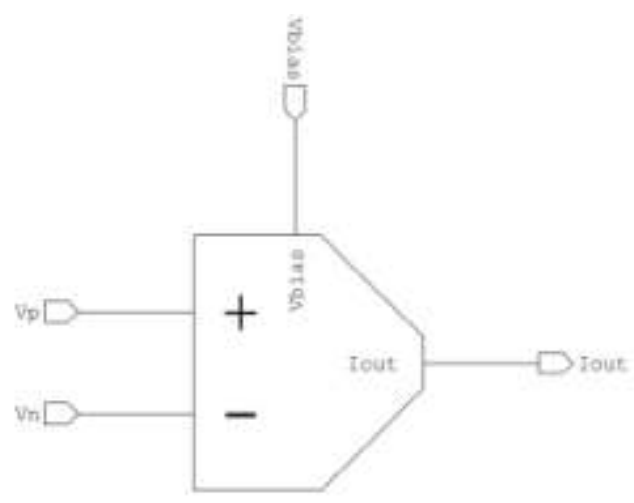

Figure 4: Symbol of OTA

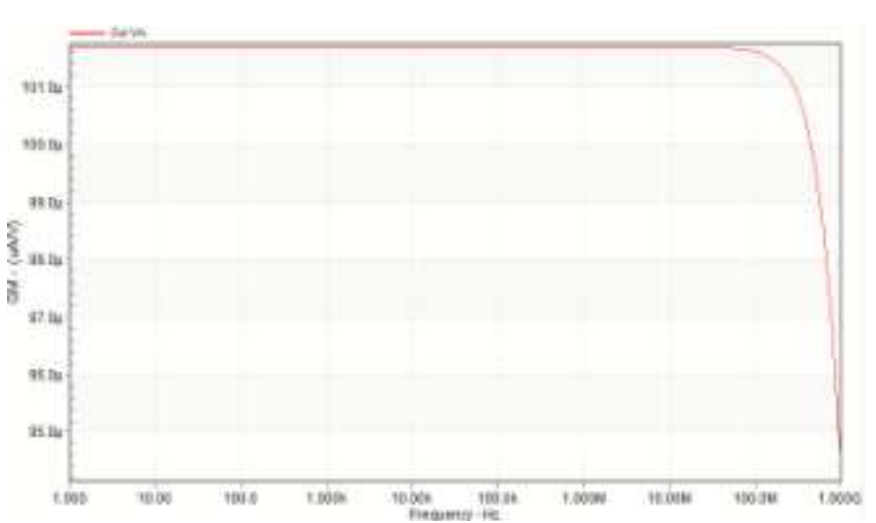

Figure 5: Variation of $G_{m}$ with frequency

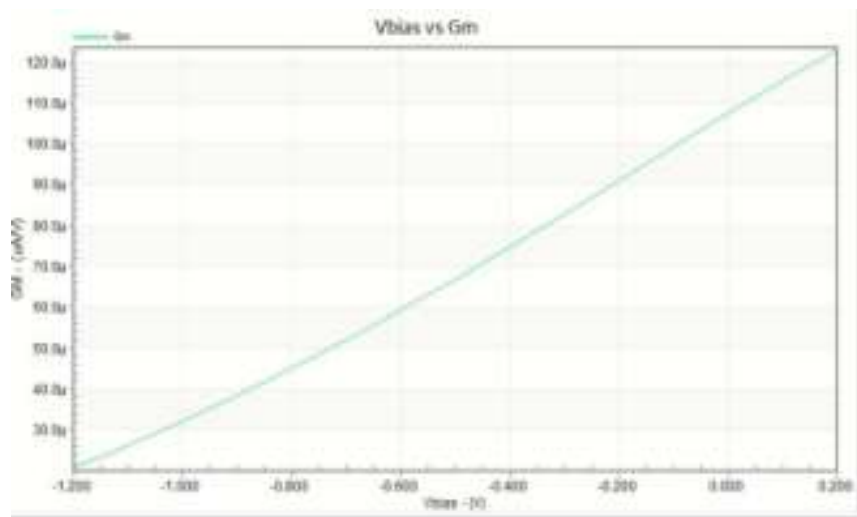

Figure 6: Variation of $\mathrm{G}$ with Vbias

\subsection{OTA-C based Comb filter}

Comb filter can also be designed by using OTA-C notch filters [9]. The circuit of comb filter using the described OTA is shown in figure 7.This filter consists of only OTAs and capacitors. The filter is modelled according to comb filter shown in figure 2.

Volume 6 Issue 12, December 2017 


\section{International Journal of Science and Research (IJSR) \\ ISSN (Online): 2319-7064}

Index Copernicus Value (2015): 78.96 | Impact Factor (2015): 6.391

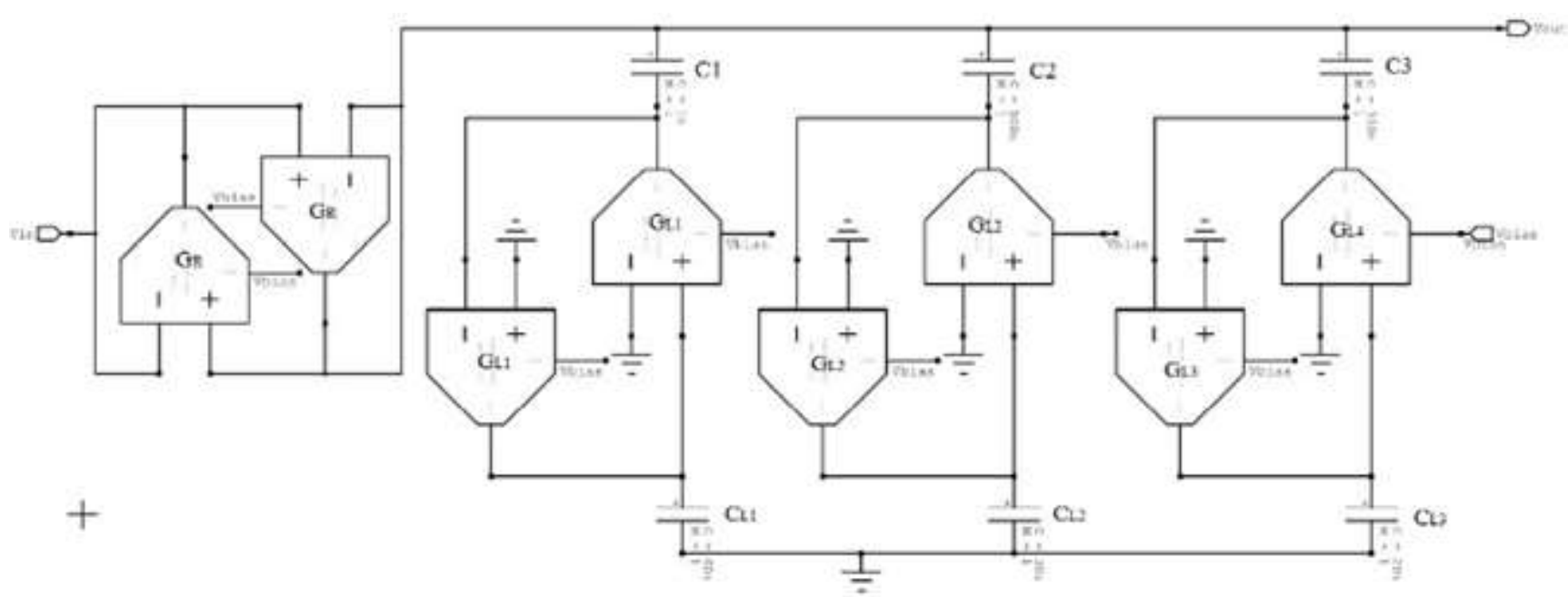

Figure 7: Comb filter using OTAs and capacitors

The inductor and resistor is modelled using OTAs, the relationship between OTA parameter $\mathrm{G}_{\mathrm{m}}$ and inductance is given as

$$
L_{1}=\frac{C_{L 1}}{G_{L 1}^{2}}
$$

and relationship between resistor and $\mathrm{G}_{\mathrm{m}}$ is given as

$$
R=\frac{1}{G_{R}}
$$

from equation (2), (3), (4), (6) and (7), filter parameters at $1^{\text {st }}$ notch frequency can be obtained as

$$
\begin{gathered}
\omega_{01}=\frac{G_{L 1}}{\sqrt{C_{L 1} C 1}} \\
Q_{01}=\frac{G_{R}}{G_{L 1}} \sqrt{\frac{C_{L 1}}{C 1}} \\
\Delta f 1=\frac{G_{L 1}{ }^{2}}{G_{R} C_{L 1}}
\end{gathered}
$$

from the above equation it can be observed that filter parameter can be tuned independently by simply adjusting the $\mathrm{G}_{\mathrm{m}}$ of individual OTAs.

The $\mathrm{G}_{\mathrm{m}}$ of all OTAs used in the comb filter is set to $78 \mu \mathrm{A} / \mathrm{V}$ by applying Vbias of $-0.4 \mathrm{~V}$. This gives value of $\mathrm{R}$ as $12.8 \mathrm{k} \Omega$. The value of capacitor $C_{L 1}$ and $C_{1}$ for notch frequency of $50 \mathrm{~Hz}$ are $60 \mathrm{nF}$ and $1000 \mathrm{nF}$ respectively. Similarly, capacitor for notch frequency $150 \mathrm{~Hz}$ and $250 \mathrm{~Hz}$ are selected as $\mathrm{C}_{\mathrm{L} 2}=$ $20 \mathrm{nF}, \mathrm{C}_{2}=350 \mathrm{nF}, \mathrm{C}_{\mathrm{L} 3}=130 \mathrm{nF}$ and $\mathrm{C}_{3}=20 \mathrm{nF}$.

\section{Results and Discussion}

The comb filter was simulated using TSPICE simulator using $250 \mathrm{~nm}$ technology. The magnitude and phase response of proposed comb filter is shown figure 8 .

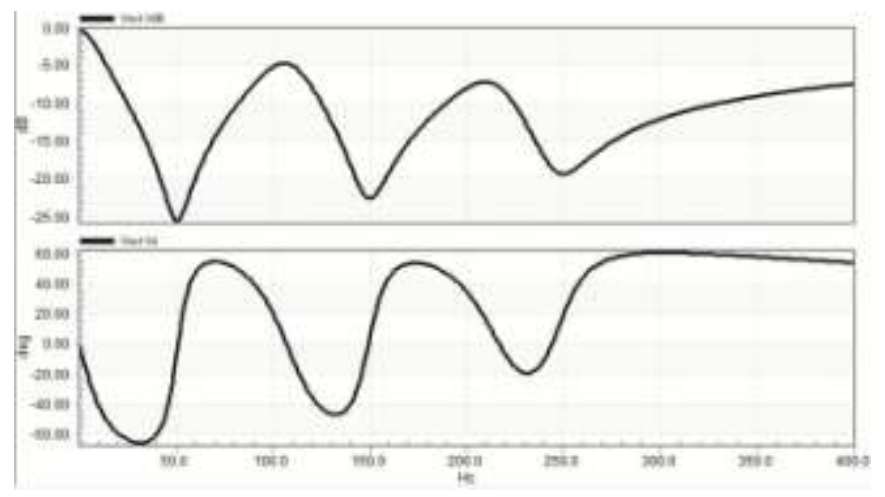

Figure 8: Magnitude and phase response of comb filter

The stopband attenuation obtained by filter at frequencies $50 \mathrm{~Hz}, 150 \mathrm{~Hz}$ and $250 \mathrm{~Hz}$ are $25 \mathrm{~dB}, 22 \mathrm{~dB}$ and $20 \mathrm{~dB}$ respectively. The THD of filter at $5 \mathrm{~Hz}$ with signal amplitude of $0.5 \mathrm{~V}$ is $1.03 \%$. The transient response of the filter for sinusoidal signal input of amplitude $1 \mathrm{~V}$ and frequency $50 \mathrm{~Hz}$, $150 \mathrm{~Hz}$ and $250 \mathrm{~Hz}$ are shown in figure 9 , figure 10 and figure 11 respectively.

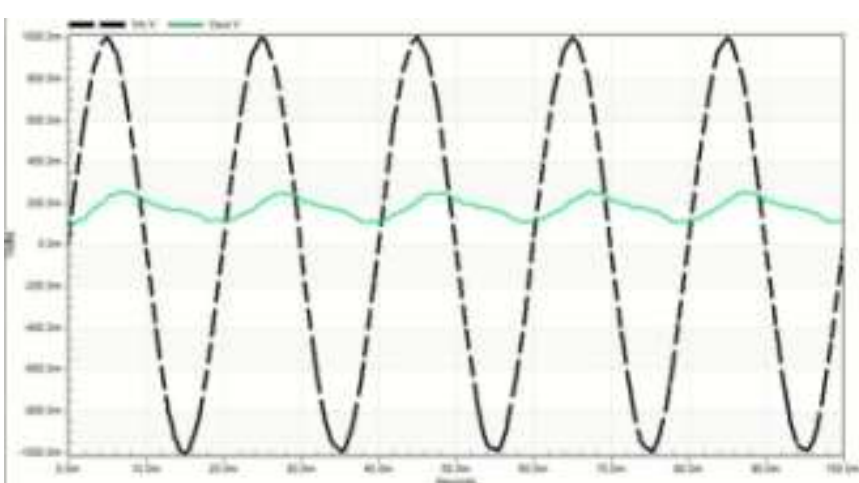

Figure 9: Filter response for $50 \mathrm{~Hz}$ sinusoidalsignal 


\section{International Journal of Science and Research (IJSR) \\ ISSN (Online): 2319-7064}

Index Copernicus Value (2015): 78.96 | Impact Factor (2015): 6.391

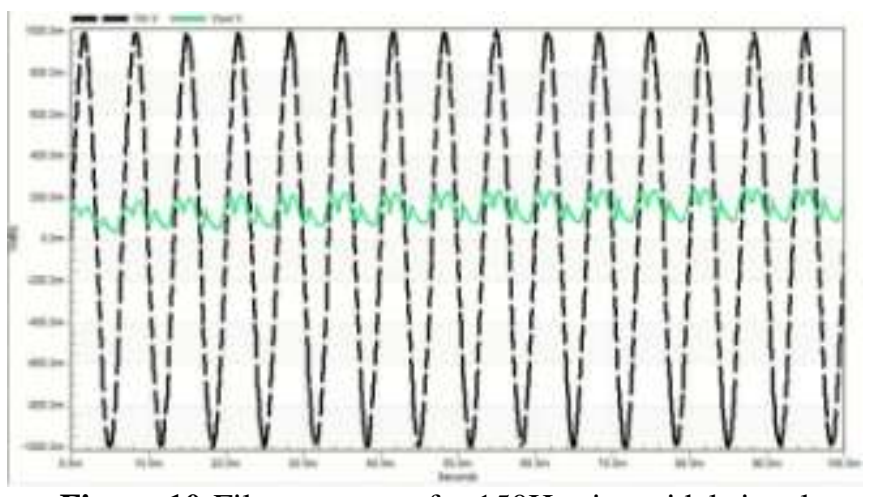

Figure 10:Filter response for $150 \mathrm{~Hz}$ sinusoidal signal

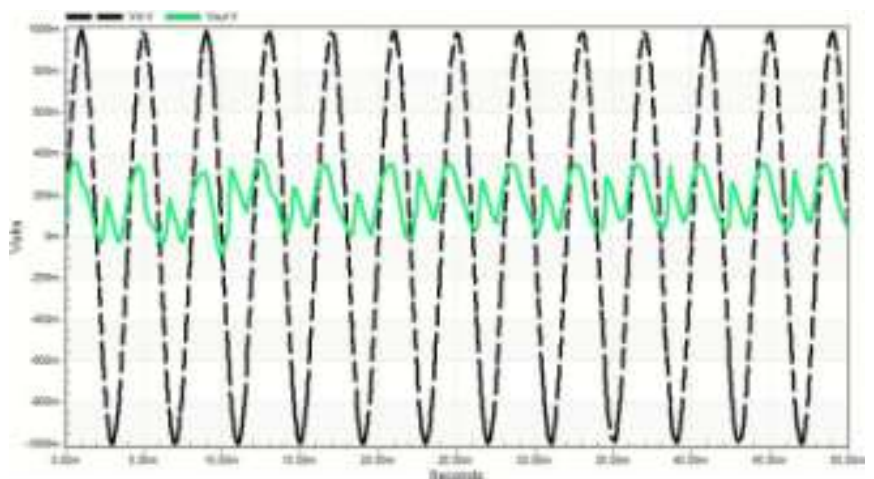

Figure 11: Filter response for $250 \mathrm{~Hz}$ sinusoidal signal

Total integrated input noise and output noise of the filter up to frequency of $100 \mathrm{~Hz}$ are $2.608 \mu \mathrm{V}$ and $403.54 \mathrm{nV}$ respectively. The average power consumption of this filter is $6.4 \mathrm{~mW}$.

\section{Conclusion and Future Scope}

An active comb filter OTA-C filter using low transconductance is proposed. The filter has been designed using 250nm CMOS technology in Tanner EDA tool and tested using TSPICE simulator.The filter performed as expected. This design has more number of active components than [4], [5] but has less number of passive components. One more advantage of OTA-C based filter design from OPAMP based design is electronic tunability of filter parameters such as center frequency and quality factor by simply controlling the bias current of OTAs. Future scope of this design can be to implement it using 14nm FinFET technology, the improvement will be lower operating voltage which will lower power consumption, more bandwidth andlower area requirement.

\section{References}

[1] J. C. Huhta and J. G. Webster, "60-Hz interference in electrocardiography," IEEE Transactions on Biomedical Engineering, vol. 20, no. 2, pp. 91-101, 1973.

[2] J. Piskorowski, "Power line interference removal from ECG signal using notch filter with non-zero initial conditions," in Proceedings of the IEEE International Symposium on Medical Measurements and Application (MeMeA '12), pp. 1-3, University of Technology, Budapest, Hungary, May 2012.
[3] Haixi Li, Jinyong Zhang, and Lei Wang, "A Fully Integrated Continuous-Time 50-Hz Notch Filter with Center Frequency Tunability," 33rd Annual International Conference of the IEEE EMBS Boston, Massachusetts USA, August 30 - September 3, 2011.

[4] C.-D. Tsai, D.-C. Chiou, Y.-D. Lin, H.-L. Chan, and C.-P. $\mathrm{Wu}$, "An active comb filter design for harmonic interference removal," Journal of the Chinese Institute of Engineers, vol. 21, no. 5, pp. 605-610, 1998.

[5] C.-T. Tsai, H.-L. Chan, C.-C. Tseng, and C.-P. Wu, "Harmonic interference elimination by an active comb filter [ECG application]," in Proceedings of 16th Annual International Conference of IEEE Engineering in Medicine and Biology Society, vol. 2, pp. 964-965, Baltimore, Md, USA, November 1994.

[6] C. Ling, P. Ye, R. Liu, and J.Wang, "A low-pass power notch filter based on an OTA-C structure for electroencephalogram," in Proceedings of the International Symposium on Intelligent Signal Processing and Communications Systems (ISPACS '07), pp. 451- 453, Xiamen, China, December 2007. Patent 3624 12, July 16, 1990.

[7] Rajeev Kumar Ranjan, Surya PrasannaYalla, ShubhamSorya, and Sajal K. Paul, "Active Comb Filter Using Operational Transconductance Amplifier," Hindawi Publishing Corporation Active and Passive Electronic Components Volume 2014, Article ID 587932, 6 pages http://dx.doi.org/10.1155/2014/587932

[8] G. Duzenlia, Y. Kcili, H. Kuntmanc, and A. Atamanb, "On the design of low-frequency filters using CMOS OTAs operating in the subthreshold region,"Microelectronics Journal, vol. 30, no. , pp. 4554, 1999.

[9] R. L. Geiger and E. S'anchez-Sinencio, "Active filter design using operational transconductance amplifiers: a tutorial," IEEE Circuits and Devices Magazine, vol. 1, no. 2, pp. 20-32, 1985.

\section{Author Profile}

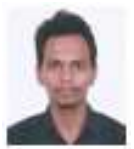

Arvind Kumar Sahureceived the B.E degree in Electronics and Telecommunications from Shri Shankaracharya Technical Campus Bhilai in 2015. He is currently in final semester of $\mathrm{M}$ Tech in VLSI Design at Shri Shankaracharya Technical Campus Bhilai. His areas of interest are biomedical signals aquisition, analog filters, wireless sensor networks. 